\title{
Investigating the Intrinsic Noise Limit of Dayem Bridge NanoSQUIDs
}

\author{
T. Patel, B. Li, J. Gallop, D. Cox, K. Kirkby, E. Romans, J. Chen, A. Nisbet and L. Hao
}

\begin{abstract}
NanoSQUIDs made from $\mathrm{Nb}$ thin films have been produced with nanometre loop sizes down to $200 \mathrm{~nm}$, using weaklink junctions with dimensions less than $60 \mathrm{~nm}$. These composite (W/Nb) single layer thin film devices, patterned by FIB milling, show extremely good low-noise performance $\sim 170 \quad n \Phi_{0}$ at temperatures between $5 \mathrm{~K}$ and $8.5 \mathrm{~K}$ and can operate in rather high magnetic fields (at least up to $1 \mathrm{~T}$ ). The devices produced so far have a limited operating temperature range, typically only 1-2 $\mathrm{K}$. We have the goal of achieving operation at $4.2 \mathrm{~K}$, to be compatible with the best SQUID series array (SSA) preamplifier available. Using the SSA to readout the nanoSQUIDs provides us with a means of investigating the intrinsic noise of the former. In this paper we report improved white noise levels of these nanoSQUIDs, enabling potential detection of a single electronic spin flip in a $1 \mathrm{~Hz}$ bandwidth. At low frequencies the noise performance is already limited by SSA preamplifier noise.
\end{abstract}

Index Terms-NanoSQUIDs, noise measurements, $T_{c}$ suppression.

\section{INTRODUCTION}

SQUIDs (Superconducting QUantum Interference Devices), macroscopic quantum sensors which have been in existence for almost 50 years, may be used for detection and measurement of a wide range of physical parameters with unequalled sensitivity. Magnetic flux is the natural quantity to which a SQUID responds directly but suitable addition of input transduction allows many other parameters such as displacement, photon detection or magnetic particle measurement to be accessed. Until recently SQUID devices were generally of relatively macroscopic size (typically from

Automatically generated dates of receipt and acceptance will be placed here. This work was supported by the UK NMS Programme, the EU EMRP project 'MetNEMS' \& SIB06, UK EPSRC and UK NIHR. The EMRP is jointly funded by the EMRP participating countries within EURAMET and the European Union. (Corresponding author: Ling Hao.)

T. Patel is with the National Physical Laboratory, Teddington, TW11 OLW, UK, e-mail: trupti.patel@npl.co.uk

B. Li is with the National Physical Laboratory, Teddington, TW11 0LW, UK, e-mail: bo.li@npl.co.uk

D.C. Cox is with the National Physical Laboratory, Teddington, TW11 0LW, UK, e-mail: david.cox@npl.co.uk.

J.C. Gallop is with the National Physical Laboratory, Teddington, TW11 0LW, UK, e-mail: john.gallop@npl.co.uk.

K. Kirkby is with University of Surrey, Guildford GU2 7XH, UK, e-mail: k.kirkby@surrey.ac.uk

J. Chen is with School of Engineering and Design, Brunel University, Uxbridge UB8 3PH, UK, e-mail: jie.chen@brunel.ac.uk.

E.J. Romans is with the London Centre for Nanotechnology, University College London, London WC1H 0AH, UK, e-mail: e.romans@ucl.ac.uk.

A. Nisbet is with University of Surrey, Guildford GU2 7XH, UK, e-mail: a.nisbett@ surrey.ac.uk

L. Hao is with the National Physical Laboratory, Teddington, TW11 0LW, UK, e-mail: ling.hao@npl.co.uk tens to a few hundred micrometres in linear dimension). Recent demonstrations show that SQUID size reductions towards the nanoscale not only retain exceptional sensitivity but, through their size, find applications in a whole new range of detection and measurement areas [1]-[6]. Flux coupling and transduction design are crucial to achieving optimal performance. To couple efficiently it is desirable to match the size of the SQUID coupling coil to the scale of the system to be measured. Although in principle an intermediate superconducting flux transformer is usual, in practice this is not feasible at the sub-micron level. Multi-turn, sub-micron coils are not readily attainable, and also the stray inductance of the leads connecting primary to secondary makes such a transformer far from optimal. Thus the present trend is to use direct coupling between the item to be measured and the nanoSQUID.

Problems arise from the use of the traditional tri-layer Josephson tunnel junctions in nanoSQUIDs. At an operating temperature $T$ the disruption from thermal fluctuations demands that the junction coupling energy exceeds these so that the critical current $I_{c} \gg k_{B} T / \Phi_{0}$ (where $k_{B}$ is Boltzmann's constant and $\Phi_{0}$ is the flux quantum $h / 2 e$ ). This condition requires $I_{c} \gg 1 \mu \mathrm{A}$. To provide such critical currents nanoscale Josephson tunnel junctions require current densities as high as $10^{9} \mathrm{~A} / \mathrm{m}^{2}$, around two orders of magnitude more than is generally achieved [7]. To circumvent this we use Dayem or weak-link 'nano-bridge' junctions prepared by focused ion beam milling of a thin superconducting film. The resulting junctions are only around $65 \mathrm{~nm}$ in length and width [8] but possess more than adequate critical current densities.

\section{DEVICE FABRICATION}

\section{A. Focussed Ion Beam (FIB) Milling of Nanobridge SQUID}

Niobium is the superconductor chosen for the nanoSQUIDs in view of its high transition temperature $(\sim 9 \mathrm{~K})$ and its small penetration depth $\left(\lambda_{0} \sim 40 \mathrm{~nm}\right)$. A thin $(150 \mathrm{~nm})$ film is sputter deposited on a Si substrate and optical lithography is used to produce a coarse pattern of strips (around $5 \mu \mathrm{m}$ wide) with larger Au wire-bonding pads.

The patterned chip is transferred to a dual-beam FIB system where a layer of amorphous tungsten is deposited over the film where the junctions are to be placed, using e-beam decomposition of $\mathrm{W}(\mathrm{CO})_{6}$. This provides both protection of the $\mathrm{Nb}$ film against $\mathrm{Ga}$ ion implantation damage and a normal metal shunt resistor for each junction. The Ga ion beam then mills away the $\mathrm{W}-\mathrm{Nb}$ bilayer to give two nanobridges within 
a)

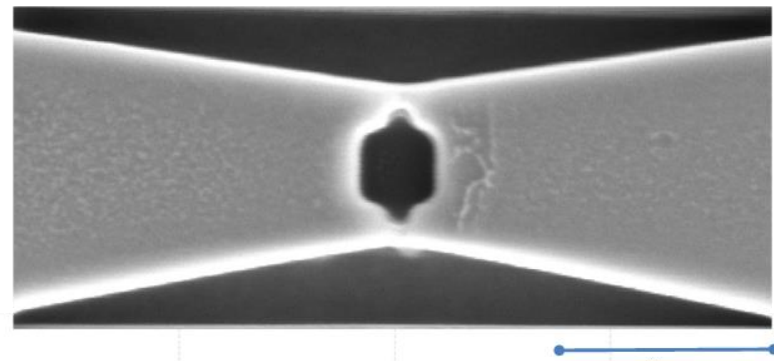

b)

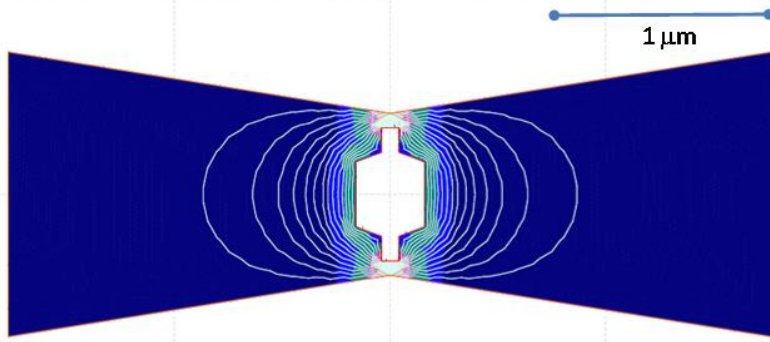

Fig. 1. In item (a), an SEM image of d.c. nanoSQUID with junctions $65 \mathrm{~nm} x$ $65 \mathrm{~nm}$ is displayed.The SQUID loop is $350 \mathrm{~nm}$ in diameter. In item (b), a plot of predicted screening supercurrent streamlines for this geometry which has a calculated inductance of $11.2 \mathrm{pH}$ is displayed.

the pre-patterned SQUID loop, typically $60-80 \mathrm{~nm}$ in both width and length. These nanoSQUIDs are highly reproducible and very stable over years [9]. They show exceptionally low flux noise $\left(<250 \mathrm{n} \Phi_{0} /(\mathrm{Hz})^{1 / 2}\right)$ [2] and can operate in high ambient magnetic fields (up to at least $1 \mathrm{~T}$ ) [10]. Fig. 1a shows an SEM image of a square loop device and fig. 1b shows a plot of the calculated screening current streamlines under application of a perpendicular magnetic field. The software package 3D-MLSI [11] has been used to calculate the loop inductance as $11.9 \mathrm{pH}$ for this geometry.

\section{NANOSQUID CHARACTERISATION}

The chosen cryogenic preamplifier is a SSA [12], essentially a current preamplifier so that the conventional current bias and voltage readout for a d.c. SQUID must be modified. It has been recognized for some years that room temperature semiconductor amplifiers have noise levels that limit the

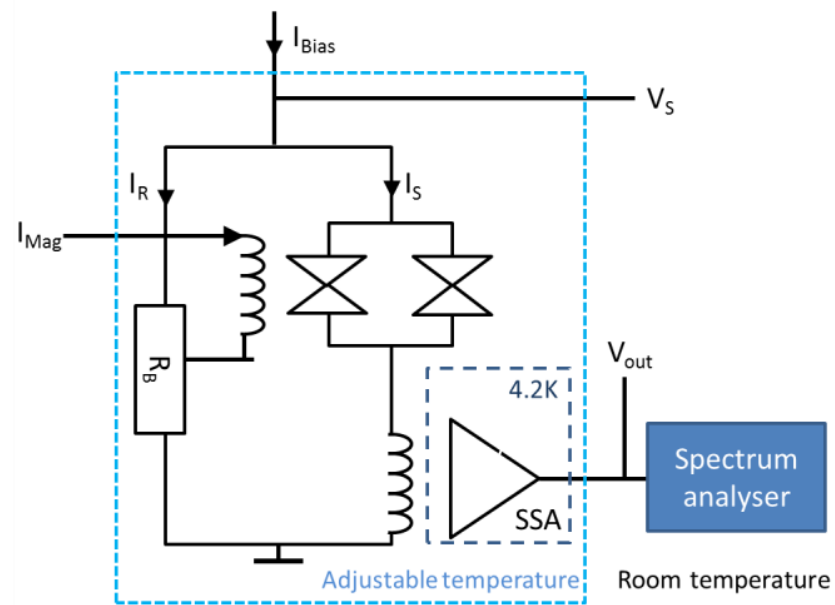

Fig. 2. Schematic of nanoSQUID bias and readout circuit showing the series SQUID array (SSA) input connection to the nanoSQUID, the coil to apply flux to it and the spectrum analyser at the SSA output to measure noise.

sensitivity of SQUIDs. Consequently cryogenic preamplifiers have been developed to try to observe the intrinsic noise limitations in SQUID detectors. We have previously reported work carried out on our nanoSQUIDs using a SQUID series array amplifier, developed at PTB [2]. In this paper we extend this investigation, presenting data which examines the improved noise performance of a nanoSQUID at elevated temperature and compare performance with modelled properties.

Figure 2 shows the schematic circuit diagram for the characterization and noise measurements. In this arrangement the SQUID is connected in parallel with a bias resistor $\boldsymbol{R}_{\boldsymbol{B}}$, typically around $0.1 \Omega$ in value. In addition the bias current, flowing through this parallel combination also flows through a small superconducting inductor coupled to the flux input of the SSA which is operated in flux-locked (i.e. linearized) mode. Note that the connections between the inductor and the nanoSQUID are made with superconducting wire and bons so there is no parasitic resistance in the SQUID bias arm. In this situation the output voltage from the SSA is directly proportional to the current flowing through this inductor. When the nanoSQUID bias current is below the critical current $I_{c}$ of the nanoSQUID all of it flows through this inductor and the output voltage from the SSA is linearly depend on bias current. However on exceeding $I_{c}$ the nanoSQUID now has a resistive component and the additional current divides between the two branches so that not all of the current flows through the inductor. The slope of the output voltage versus bias current will now change By subtracting a constant slope from the $V_{S S A}$ output voltage versus bias current plots it is possible to convert the SSA readout voltage into a conventional current voltage characteristic (IVC) of the d.c. nanoSQUID. A family of such curves for a range of magnetic flux values applied to the nanoSQUID loop is shown in fig. 3 where the flux ranges over a range of more than two flux quanta $\left(2 \Phi_{0}\right)$.

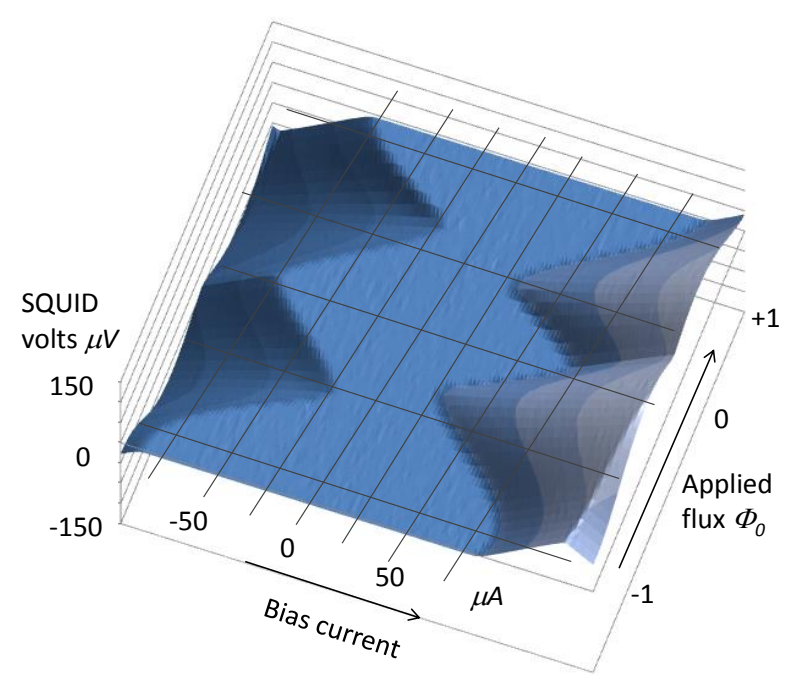

Fig. 3. SQUID output voltage versus bias current of nanoSQUID, corrected for fixed slope corresponding to d.c. supercurrent. Different IVCs are shown for a range of applied magnetic flux values. Note that the ratio of maximum critical current to minimum critical current is 3.5 
Note that the flux to current transfer function $d I / d \Phi$ is as high as $1.9 \mathrm{~mA} / \Phi_{0}$. The gain (or transfer function) for either a voltage preamplifier $d V / d \Phi$ or a current preamplifier $d I / d \Phi$ may be calculated from this diagram by taking cuts at constant bias current or constant SQUID voltage respectively. It is clear from the overall shape that to maximize both values bias currents just greater than the SQUID critical current will achieve this, though of course this may limit the open loop dynamic range. Fig. 4 illustrates a typical horizontal cut for a nanoSQUID with $300 \mathrm{~nm}$ diameter loop, operated at $7.45 \mathrm{~K}$. The total range of applied flux corresponds to $2 \Phi_{0}$. The flat regions close to $0 \Phi_{0}$ and $-1 \Phi_{0}$ correspond to regions where the critical current of the SQUID is just greater than the bias current.

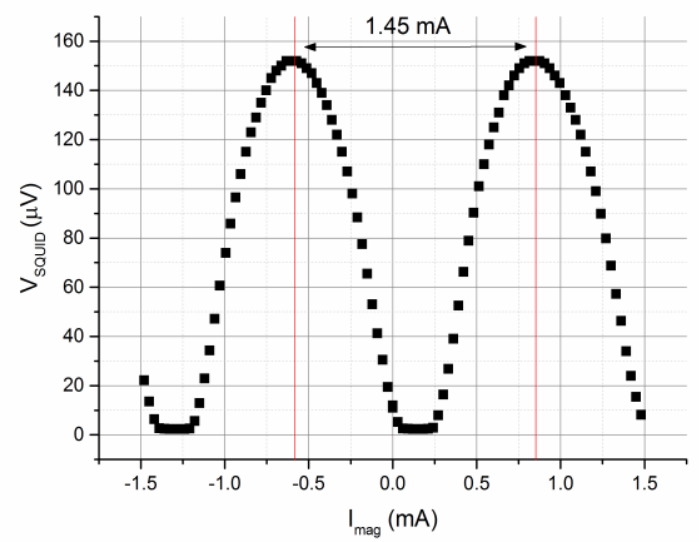

Fig. 4. Plot of nanoSQUID output voltage $V_{S Q U I D}$ versus current through magnet $I_{\text {mag. }}$.

\section{SQUID NOISE MEASUREMENTS USING SSA PREAMPLIFIER}

The SSA operates at a fixed temperature of around $4.2 \mathrm{~K}$. The current noise, referred to the input inductor, in the white noise range above around $100 \mathrm{~Hz}$ is $10 \mathrm{pA} /(\mathrm{Hz})^{1 / 2}$. In contrast the FIB milled nanoSQUID, contained within a vacuum can and only very weakly thermally coupled to the liquid helium bath, is operated at an adjustable temperature. A heater and temperature sensor allow control with sub-mK stability without perturbing the SSA operating parameters. Having selected a control temperature and nanoSQUID bias current the magnetic field applied to the nanoSQUID loop is adjusted to provide optimize the magnetic flux to nanoSQUID voltage transfer function $d V_{\text {SQUID }} / d \Phi_{x}$. This is achieved by sweeping a current $(<2 \mathrm{~mA})$ through a small superconducting magnet which applies magnetic field perpendicular to the nanoSQUID loop. A typical plot of $V_{S Q U I D}$ versus $\Phi_{x}$ is shown in Fig. 4. Having selected a value for $\Phi_{x}$ which corresponds to maximum value for $\left|d V_{S Q U I D} / d \Phi_{x}\right|$ the output voltage from the room temperature amplifier of the SSA is fed to a low frequency spectrum analyser. The noise spectra between 0.03 $\mathrm{Hz}$ and $10^{5} \mathrm{~Hz}$ are sampled 1000 times, averaged and recorded. Knowing the gain of the system it is then straightforward to convert the observed noise to the square root of power spectral density of magnetic flux noise $S_{\Phi}{ }^{1 / 2}$ referred to the input at the nanoSQUID. A summary of these noise plots is shown in the following section.

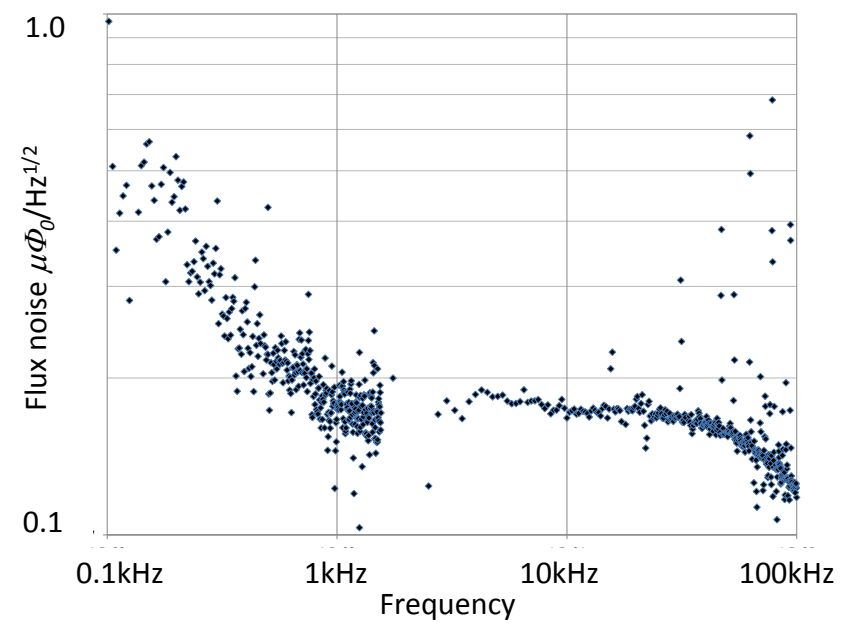

Fig. 5 Power spectral density $\left(S_{\Phi}\right)^{1 / 2}(f)$ of the nanoSQUID shown in Fig.4, operating at $7.45 \mathrm{~K}$

\section{NANOSQUID NOISE ANALYSIS}

The voltage noise is measured at the output of the flux locked SSA preamplifier and fed to a digital spectrum analyser, as shown in fig. 2. The power spectral density $S_{v}(f)$ is measured by the spectrum analyser and may be converted to effective flux noise at the input to the nanoSQUID using the following relationship:

$$
S_{\Phi}^{1 / 2}=V_{n} \frac{\partial I_{S S A}}{\partial V_{o}} \frac{\partial \Phi}{\partial I_{S S A}}
$$

The second and third factors on the right hand side of equation (1) can be measured from the data as displayed in Fig. 3. The result is shown in Fig. 5, a typical noise spectrum from a nanoSQUID, of the type shown in an SEM image of Fig.1. The data points represent the full noise data corrected for the SSA noise when the nanoSQUID is unbiased. Noise figures are subtracted in quadrature, in the conventional way. Below about $100 \mathrm{~Hz}$ it is not possible to carry out the quadrature subtraction because the noise level in the biased nanoSQUID is indistinguishable from the noise from the unbiased system. The implications of this are discussed in the following section.

\section{CONCLUSIONS}

We show in this paper that the nanoSQUIDs fabricated by FIB milling of single layer $\mathrm{Nb}$ thin film using Dayem microbridge junctions show even better noise performance than we have previously reported. The exceptional white flux noise figures demonstrate the efficacy of the technology. A large number of other similar devices show similar performance, indicating that the process is repeatable. A point of particular interest is the degree of critical current modulation produced by a flux signal, as mentioned in the caption to Fig. 3. The variation between maximum and minimum critical currents shows that the dimensionless critical current modulation parameter $\Delta I_{c}, \frac{\Delta I_{c}}{I_{c \max }}>0.78$. This is attained at a temperature of $7.45 \mathrm{~K}$ and a value of $I_{\text {cmax }}=$ $75 \mu \mathrm{A}$. This can only be achieved if two separate conditions 
apply. First, the McCumber shielding parameter $\beta_{L}=$ $2 L I_{\text {cmax }} / \Phi_{0}$ must be less than 1.0 (see for example Chapter 2 in ref. [13]). This sets an upper limit of $13 \mathrm{pH}$ on the total SQUID loop inductance which compares well with the calculation of $L$ made using the 3D-MLSI software shown in Fig. 1(b). This is significant in that it confirms that geometrical inductance calculations can provide realistic estimates for Dayem junction SQUID properties and the kinetic inductance contribution from the junctions, which would tend to reduce the SQUID response, can be neglected under these conditions.

The second condition relates to the similarity of the critical currents of the individual Dayem microbridges of the SQUID. From the observed high value of the current modulation depth the dimensionless difference $\Delta i_{c}=\left|I_{c l}-I_{c 2}\right| /\left(I_{c l}+I_{c 2}\right)$ between the individual junction critical currents must be less than 0.22 . Thus these junctions are particularly well matched which is a tribute to the precision of FIB Milling and also the uniformity of the $\mathrm{Nb}$ thin film. If a grain boundary existed within one microbridge but not the other it is very improbable that theoretical critical currents would be so well matched. The high value of $\Delta i_{c}$ also implies that the microbridges are smaller in width and length on the scale of the superconducting cohenernce length $[14,15]$

It is also interesting to estimate the energy sensitivity $\varepsilon_{n}$ of these nanoSQUIDs, defined by

$$
\varepsilon_{n}=\frac{S_{\Phi}}{2 L}
$$

Using the observed white noise value of $S_{\Phi}=3 \times 10^{-14} \Phi_{0}{ }^{2}$ and the calculated/estimated inductance of $11.2 \mathrm{pH}$ we reach a value of $4.6 \times 10^{-33} \mathrm{~J} / \mathrm{Hz}$ or around $45 \mathrm{\hbar}$. Since the standard zero point energy fluctuation limit is still far from achieved further developments may be expected to permit even better performance at these relatively elevated temperatures. It should be noted that the noise level is already sufficient to attain the previously stated goal of achieving a $1 \mathrm{~Hz}$ bandwidth detection capability of sensing the magnetic moment reversal of a single Bohr magneton. This assumes that the spin is oriented in the plane of the nanoSQUID loop and in the nearfield regime, positioned close to one junction at the perimeter of the loop For details of the calculation see [15-17].

The fact that the low frequency noise (for $f$ less than typically $100 \mathrm{~Hz}$ ) is still limited by the SSA readout suggests that in this $1 / f$ range the nanoSQUID may have superior performance to the trilayer junction based SSA preamplifier. We speculate that this may be due to an absence of two level fluctuators in the nanobridge devices, since the presence of these in trilayer oxide barriers are believed to be responsible for low frequency excess noise. If this is the case and, given the reproducibility of the Dayem bridge junctions, it may be useful to try to develop a series SQUID array system based on microbridge junction technology.

The main restriction on the performance of these weak-link
SQUIDs is that they have a limited range of operating temperatures, typically from $6 \mathrm{~K}$ to $8.5 \mathrm{~K}$. We have demonstrated that it is possible to suppress the range of operating temperature to below $4.2 \mathrm{~K}$ and examining the noise levels in these suppressed $T_{c}$ devices is the subject of some current research.

Future work will concentrate on developing nanoSQUIDs using this technology which are physically and geometrically matched to the entities that they are designed to detect and measure whether these are nanomagnetic particles [18], nanoelectromechanical resonators [19] or single energetic particles [20]. Perhaps the most novel of these is the combination of nanoSQUIDs with matched nanomechanical resonators. Fig. 6 shows a new example of a slot shaped nanoSQUID aimed at providing sensitive detection of the vibration of such a double clamped beam nanocantilever.

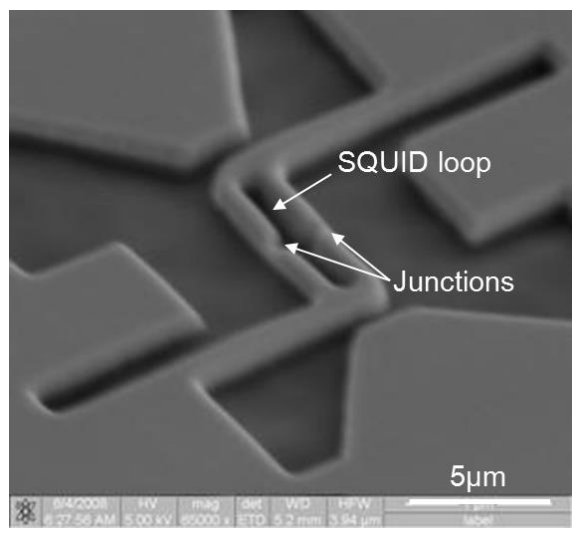

Fig. 6. SEM images of the new slot-shaped nanoSQUID structure $(100 \mathrm{~nm} \times$ $900 \mathrm{~nm}$ ) designed for optimum coupling to a double clamped nanoelectromechanical system (NEMS) beam resonator.

\section{REFERENCES}

[1]. C P Foley and H Hilgenkamp, "Why nanoSQUIDs are important", Supercond. Sci. Technol. vol. 22 064001, (2009)

[2]. L. Hao, J. C. Macfarlane, J. C. Gallop, D. Cox, J. Beyer, D. Drung, and T. Schurig, "Measurement and noise performance of nanosuperconducting-quantum interference devices fabricated by focused ion beam”, Appl. Phys. Lett., vol. 92, p. 192507 (2008)

[3]. David Tilbrook, "NanoSQUID sensitivity for isolated dipoles and small spin populations", Superconduct. Sci. Technol. vol. 22 064003 (2009)

[4]. R Russo, C Granata, E Esposito, D Peddis, C. Cannas and A. Vettoliere, "Nanoparticle magnetization measurements by a high sensitive nano-superconducting quantum interference device" Appl. Phys. Lett. vol. 101, 122601 (2012)

[5]. C. Granata et al, "Three-dimensional spin nanosensor based on reliable tunnel Josephson nano-junctions for nanomagnetism investigations" Appl. Phys. Lett. vol. 103, 102602 (2013)

[6]. R. Wolbing et al., "Nb nano superconducting quantum interference devices with high spin sensitivity for operation in magnetic fields up to 0.5 T", Appl. Phys. Lett. vol. 102, 192601 (2013)

[7]. Vincenzo Lacquaniti, Sabino Maggi, Eugenio Monticone, and Raffaella Steni, "Properteis of r.f. sputtered Josephson SNAP junctions" IEEE Trans. Appl. Supercond. vol. 6, 24-31 (1996)

[8]. L Hao, "Quantum Detection Applications of NanoSQUIDs fabricated by Focussed Ion Beam", J. Phys.: Conf. Ser. 286, 012013 (2011) 
[9]. L. Hao, J. C. Macfarlane, J. C. Gallop, D. Cox, D. Hutson, P. Josephs-Franks, J. Chen, and S. K. H. Lam, "Novel methods of fabrication and metrology of superconducting nanostructures," IEEE Trans. Instrum.and Meas., vol. 56, pp. 392-396 (2007)

[10]. E. J. Romans, S. Rozhko, L. Young, A. Blois, L. Hao, D. Cox, J. C. Gallop, "Noise Performance of Niobium Nano-SQUIDs in Applied Magnetic Fields", IEEE Trans. Appl. Supercond., vol.21, pp. 404-7 (2011)

[11]. M M Khapaev, 3D-MLSI- The program for extraction of 3D inductances of multilayer superconductor circuits. [online]. Available: http://vm.cs.msu.ru/sotr/vmhap/3dmlsi/3dmlsm.pdf

[12]. D. Drung, C. Aßmann, J. Beyer, A. Kirste, M. Peters, F. Ruede, and T.Schurig, IEEE Trans. Appl. Supercond. vol. 17, 699 (2007).

[13]. J. Clarke and A. Braginski, 'The SQUID Handbook', Wiley-VCH Verlag (2005)

[14]. C. Granata at al "Noise theory of dc nano-SQUIDs based on Dayem nanobridges", Phys Rev. B, vol, 84, pp. 224516, (2011)

[15]. L. Hao, D.C. Cox, J.C. Gallop, J. Chen, "Fabrication and Analogue Applications of nanoSQUIDs using Dayem Bridge Junctions", IEEE JST Quantum Electronics, 10.1109/JSTQE.2014.2354634

[16]. V. Bouchiat, "Detection of magnetic moments using a nanoSQUID: limits of resolution and sensitivity in near-field SQUID magnetometry", Supercon. Sci. Technol. vol. 22, 064002 (2009)

[17]. J. C. Gallop, "SQUIDs : some limits to measurement", Supercond. Sci. Technol. vol. 16 pp 1575-82 (2003)

[18]. L. Hao, C. Aßmann, J. C. Gallop, D. Cox, F. Ruede, O. Kazakova, P. Josephs-Franks, D. Drung, and Th. Schurig "Detection of single magnetic nanobead with a nano-superconducting quantum interference device", Appl. Phys. Lett. vol. 98092504 (2011)

[19]. L. Hao, D. C. Cox, J. C. Gallop, E. J. Romans, J. C. Macfarlane, and J. Chen, "Focused ion beam nanoSQUIDs as novel NEMS resonator readouts", IEEE Trans. Appl. Supercond., vol. 19, pp. 693-696 (2009)

[20]. S. Galer, L. Hao, J. Gallop, H. Palmans, K. Kirkby and A. Nisbet, "Design concept for a novel SQUID based microdosimeter", MICROS 2009 Symposium Proceedings, Special issue of Radiation Protection Dosimetry, Oxford Journals , Oxford University Press (2010) 